\title{
The prognostic and predictive significance of cytokeratin 5/6 and epidermal growth factor receptor in metastatic triple-negative breast cancer treated with maintenance capecitabine
}

\author{
Yiping Zhu ${ }^{1+\wedge}$, Kai Li ${ }^{2 *}$, Jieling Zhang ${ }^{2 \#}$, Lu Wang ${ }^{1}$, Lili Sheng ${ }^{1}$, Liang Yan $^{2}$ \\ ${ }^{1}$ Department of Oncology, The First Affiliated Hospital of Wannan Medical College, Wuhu, China; ${ }^{2}$ Wannan Medical College, Wuhu, China \\ Contributions: (I) Conception and design: Y Zhu, L Yan; (II) Administrative support: L Sheng; (III) Provision of study materials or patients: Y Zhu, L \\ Wang, L Sheng; (IV) Collection and assembly of data: K Li, J Zhang; (V) Data analysis and interpretation: Y Zhu, K Li, J Zhang; (VI) Manuscript \\ writing: All authors; (VII) Final approval of manuscript: All authors. \\ \#These authors contributed equally to this work. \\ Correspondence to: Lili Sheng. Department of Oncology, The First Affiliated Hospital of Wannan Medical College, No. 2 Zheshan West Road, \\ Jinghu District, Wuhu 241000, China. Email: 13605535185@163.com; Liang Yan. Department of Biochemistry, Wannan Medical College, No. 22 \\ Wenchang West Road, Yijiang District, Wuhu 241000, China. Email: yane2002@126.com.
}

\begin{abstract}
Background: Capecitabine is the most widely used agent for maintenance chemotherapy in patients with metastatic triple-negative breast cancer (mTNBC). However, there are no biomarkers for identifying mTNBC patients who could benefit from capecitabine maintenance.

Methods: The prognostic roles of cytokeratin 5/6 (CK5/6), epidermal growth factor receptor (EGFR), and maintenance therapy were evaluated in mTNBC patients. Both CK5/6 and EGFR were detected using immunohistochemistry. Of 115 patients who achieved disease control, 56 received capecitabine maintenance therapy and 59 underwent observation. The progression-free survival (PFS) and overall survival (OS) of the patients were evaluated.
\end{abstract}

Results: The median PFS and OS were longer in the maintenance group than that in the observation group (7.3 versus 5.7 months, $\mathrm{P}=0.0016 ; 22.4$ versus 17.9 months, $\mathrm{P}=0.0055$ ). Patients with basal-like TNBC had a poorer survival times than in those with non-basal-like TNBC $(\mathrm{P}=0.0062)$. Capecitabine maintenance significantly prolonged the OS of non-basal-like TNBC patients $(\mathrm{P}=0.0257)$, while in the basal-like TNBC patients, the difference was not significant $(\mathrm{P}=0.0541)$. Multivariate analysis revealed that the prolonged OS was related to age $>50$ years $(\mathrm{P}=0.005)$, presence of visceral metastases $(\mathrm{P}=0.035)$, response to initial therapy $(\mathrm{P}=0.017)$, maintenance therapy $(\mathrm{P}=0.033)$, and CK5/6 and EGFR status $(\mathrm{P}=0.032)$. Compared with the observation group, toxicities of all grades were more frequently observed in the maintenance group, including neutropenia, $85.71 \%$ vs. $25.87 \%, \mathrm{P}<0.001$; thrombocytopenia, $55.36 \%$ vs. $11.86 \%, \mathrm{P}<0.001$; anemia, $82.14 \%$ vs. $52.54 \%, \mathrm{P}=0.001$; nausea $83.47 \%$ vs. $11.86 \%, \mathrm{P}<0.001$; vomiting $69.64 \%$ vs. $8.47 \%$, $\mathrm{P}<0.001$; and hand-foot syndrome (HFS) $32.14 \%$ vs. $1.69 \%, \mathrm{P}<0.001$.

Conclusions: Our study revealed that patients with non-basal-like TNBC had a better clinical outcome than those with basal-like TNBC, and capecitabine maintenance treatment significantly prolonged PFS and OS in patients with TNBC. Patients with non-basal-like TNBC could benefit from maintenance therapy with capecitabine and CK5/6 and EGFR are biomarkers for TNBC prognosis.

Keywords: Maintenance chemotherapy; capecitabine; triple-negative breast cancer (TNBC)

Submitted Apr 03, 2020. Accepted for publication Jan 27, 2021.

doi: $10.21037 /$ tcr-20-1760

View this article at: http://dx.doi.org/10.21037/tcr-20-1760

$\wedge$ ORCID: 0000-0002-4738-1125. 


\section{Introduction}

Breast cancer is the most common female malignancy worldwide. In approximately $5 \%$ of cases, the breast cancer has metastasized by the time of initial diagnosis. Despite most operable patients receiving surgery and adjuvant chemotherapy, the rate of recurrence or metastasis reaches $20-35 \%$ (1). Metastatic breast cancer (MBC) is an incurable disease, and the major goal of treatment is to relieve and control patients' symptoms, improve their quality of life, and prolong their survival time (2).

Based on gene and immunohistochemistry analyses, breast cancers can be divided into different subtypes. Biological characteristics and clinical outcomes differ among patients with different subtypes, and the treatment strategies also vary. Expert consensus recommends endocrine therapy as the treatment of choice for hormone receptor (HR)positive tumors. Trastuzumab is also used in combination with chemotherapy and then maintained on its own in HER2-positive patients. Triple-negative breast cancer (TNBC), which is defined as HER2-negative and HRnegative, accounts for nearly $12-17 \%$ of all breast cancers (3). TNBC progresses rapidly and is life threatening, and chemotherapy is recommended as treatment (4-6).

Compared with HER2/HR-positive breast cancers, TNBC is more prone to recurrence and metastasis, even with similar treatments, and is associated with shorter progression-free survival (PFS) and overall survival (OS). The median time to recurrence for metastatic triple-negative breast cancer (mTNBC) patients is $1-2$ years, and the median OS (mOS) is almost 1 year $(7,8)$. Lehmann et al. compiled the gene expression profiles of 587 TNBC patients from 21 independent studies and identified 6 different TNBC subtypes using cluster analysis (9). These subtypes consist of basal-like 1, basal-like 2, immunomodulatory (IM), mesenchymal (M), mesenchymal stem cell-like (MSL), and lumen androgen receptor (LAR) breast cancer. Different subtypes present with unique gene expression profiles and are associated with different signaling pathways. Of the 6 subtypes, basal-like breast cancer (BLBC) has attracted the most attention. The majority of BLBCs exhibit a triple-negative phenotype and have a poor prognosis (10). Nielsen et al. examined the protein expression patterns in various basal-like breast tumors (11). They reported that the detection of cytokeratin 5/6 (CK5/6) in TNBC could accurately identify BLBC and showed high specificity. Rakha et al. (12) reported that CK5/6-positive and epidermal growth factor receptor
(EGFR)-positive patients had more BRCA1 mutations, more distant metastases, and a poor prognosis compared with CK5/6- and EGFR-negative patients.

Capecitabine is widely used in the treatment of breast cancer. The FinXX study (13) and the CSCSG-010 study (14) showed that capecitabine-based adjuvant chemotherapy significantly prolonged the recurrence-free survival and OS of TNBC patients, while the GEICAM/ 2003-11 (15) study showed that patients with a non-basallike phenotype could benefit from the addition of extended capecitabine treatment in early TNBC. In mTNBC, capecitabine maintenance therapy has been reported to demonstrate high activity and manageable safety $(16,17)$. Clinical biomarkers to predict the efficacy of capecitabine are extremely important in TNBC, although studies in this area are relatively limited.

This retrospective cohort study focused on the correlations between CK5/6 and EGFR expression, the prognosis of TNBC, and the efficacy of capecitabine maintenance in patients with different TNBC subtypes. We present the following article in accordance with the REMARK reporting checklist (available at http://dx.doi. org/10.21037/tcr-20-1760).

\section{Methods}

\section{Patients}

Between January 2012 and December 2016, 164 mTNBC patients received first-line combination chemotherapy in the Oncology Department of the First Affiliated Hospital of Wannan Medical College, Anhui, China. Table 1 shows the baseline characteristics of the patients. All patients were aged 18 years or above, and all had confirmed recurrent or metastatic TNBC. Of 115 patients who achieved disease control after first-line combination chemotherapy, 93 had received an anthracycline-containing regimen, and 85 had received a taxane-containing regimen as adjuvant/ neoadjuvant therapy. All patients had a good Eastern Cooperative Oncology Group (ECOG) score and a life expectancy of $\geq 3$ months, along with at least 1 measurable metastatic lesion detected by computed tomography or magnetic resonance imaging examination, and adequate organ function.

Patients with clinically detectable meningeal and/or brain parenchyma metastases, congestive heart failure, or reduced hepatic or renal function were excluded, as were those with HER2- or HR-positive cancers. We also excluded patients 
Table 1 Characteristics of 115 mTNBC patients treated with gemcitabine combined with cisplatin

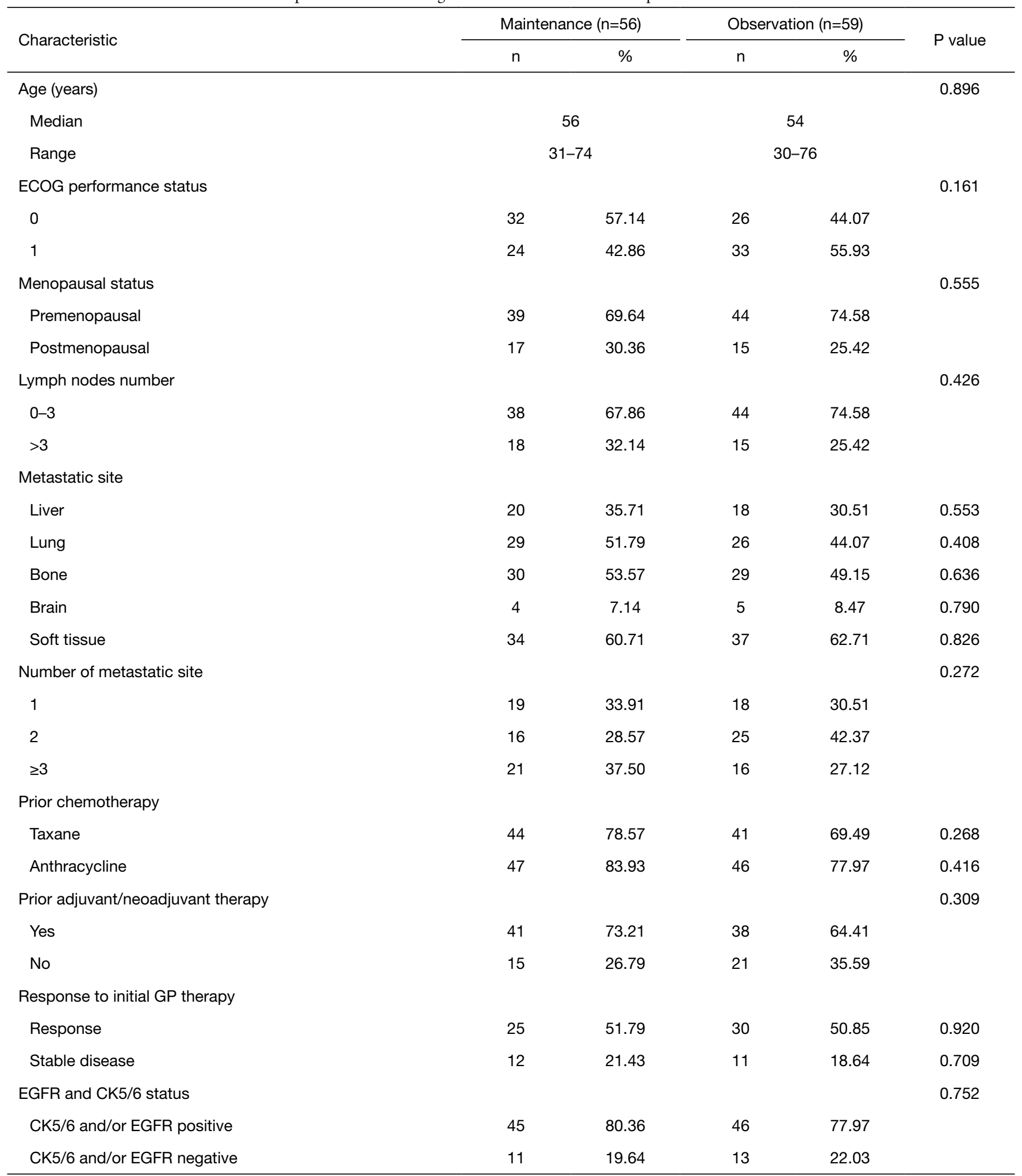

ECOG PS, Eastern Cooperative Oncology Group performance status; mTNBC, metastatic triple-negative breast cancer; EGFR, epidermal growth factor receptor. 
with concurrent tumors and those who had been diagnosed with tumor within the previous 5 years, as well as pregnant or breast-feeding women. The study was conducted in accordance with the Declaration of Helsinki (as revised in 2013) and was approved by the Ethical Committee of the First Affiliated Hospital of Wannan Medical College (No. 2008-7). Individual consent for this retrospective analysis was waived.

\section{Study design}

In this retrospective study, 115 of 164 patients achieved disease control, of whom 56 received capecitabine maintenance chemotherapy and 59 received observation. Patients in the maintenance group received capecitabine $\left(1,000 \mathrm{mg} / \mathrm{m}^{2}\right)$ orally bid for 2 weeks every 3 weeks along with metoclopramide and dexamethasone to prevent nausea and vomiting, until disease progression, intolerable toxic effects, or withdrawal of consent. In the observation group, patients received observation until disease progression or withdrawal of consent. Laboratory assessments, such as routine blood counts, serum liver function markers, creatinine, and tumor biomarker levels were carried out at the beginning of each cycle. The tumors were measured at baseline and every 2 cycles through computed tomography scanning, magnetic resonance imaging, ultrasound, or physical examinations. Efficacy and toxicity were evaluated every 2 cycles.

\section{Immunobistochemistry}

Tumor tissues from TNBC patients were fixed in formalin, embedded in paraffin, and cut to a thickness of $4 \mu \mathrm{m}$. The sections were baked and dewaxed, and the endogenous catalase was removed. After blocking, the sections were incubated with primary antibody targeting CK5/6 (1:250, MA5-12429, Thermo Fisher Scientific, France) and EGFR (1:250, MA5-13070, Thermo Fisher Scientific, France) overnight at $4{ }^{\circ} \mathrm{C}$, then incubated with secondary horseradish peroxidase (HRP)-conjugated antibody (Cell Signaling Technology, Danvers, MA, USA). Sections were then stained with 3,3'-diaminobenzidine. Two independent pathologists scored the results. All sections were observed under fluorescence microscopy. The percentage of positive cells in 5 random high power fields (HPFs) and the intensity of positive staining were evaluated.

\section{Statistical analysis}

The primary endpoint of the study was PFS, and clinical efficacy and OS were the secondary endpoints. Response evaluation criteria for solid tumors (RECIST version 1.1) (18) was used to evaluate clinical efficacy. SPSS 19.0 software was used to analyze all data. The Kaplan-Meier estimator and log-rank test were used to analyze median PFS (mPFS) and mOS, and a Cox regression model was used to analyze the effects of age, menopausal status, metastatic sites, prior chemotherapy status, and ECOG. $\mathrm{P}<0.05$ in a 2 -sided test was considered to represent a statistically significant difference.

\section{Results}

\section{Clinicopathological features}

After the first 6 cycles of combination chemotherapy, 115 patients $(70.12 \%, 115 / 164)$ had achieved disease control. Subsequently, 56 patients received maintenance therapy with capecitabine and 59 patients underwent observation alone. The maintenance group included 39 premenopausal women, and the observation group included 44 premenopausal women. The median ages in the maintenance and observation groups were 56 years and 54 years, respectively. All patients had an ECOG score of $0-1$, and most patients in both groups had multiple metastatic sites and visceral metastases. More than $80 \%$ of the patients underwent surgical resection and received adjuvant/neoadjuvant chemotherapy. CK5/6 and EGFR were negatively expressed in nearly $20 \%$ of cases (Figure 1 ). The clinicopathological features of the patients are shown in Table 1.

\section{Efficacy analysis}

In the 164 patients investigated, the objective response rate and the disease control rate after the first 6 cycles of combination therapy were $51.83 \%(85 / 164)$ and $70.12 \%$ $(115 / 164)$, respectively. As of the deadline for follow-up (August 31, 2019), 9 patients in the maintenance group were still alive, and 4 were alive in the observation group. In the maintenance group, the median follow-up time was 29.8 months, the mPFS was 7.3 months, and the mOS was 22.4 months. In the observation group, the median followup time was 26.1 months, the mPFS was 5.7 months, and the mOS was 17.9 months. Maintenance therapy significantly prolonged the mPFS time by 1.6 months $(\mathrm{P}=0.0016)$ (Figure 2$)$ and the mOS time by 4.5 months $(\mathrm{P}=0.0055)$ (Figure 3A). For all 115 patients, those with 

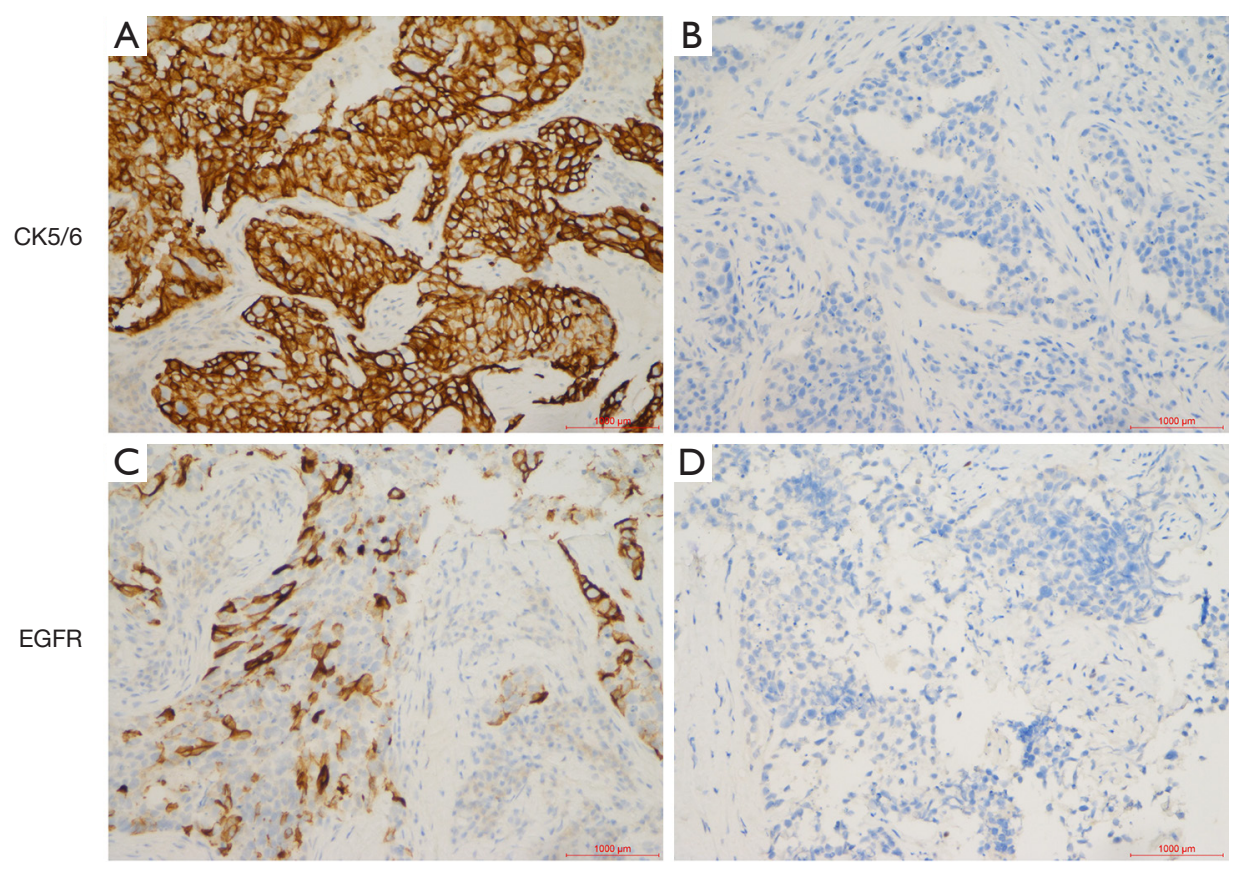

Figure 1 CK5/6 and EGFR expression by immunohistochemistry. (A,B) CK5/6 positive/negative staining; (C,D) EGFR positive/negative staining. EGFR, epidermal growth factor receptor.

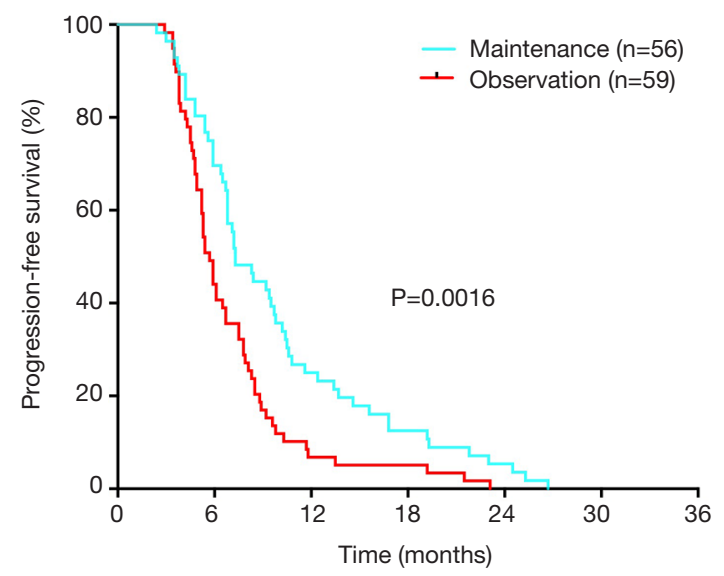

Figure 2 Progression-free survival (PFS) of TNBC patients treated with combination chemotherapy followed by capecitabine maintenance and observation. TNBC, triple-negative breast cancer.

basal-like TNBC had a shorter survival time than those with non-basal-like TNBC (18.6 vs. 27.4 months, $\mathrm{P}=0.0062$ ) (Figure 3B). In the maintenance group, the OS of non-basallike TNBC patients was 9.4 months longer than that of basal-like TNBC patients (30.2 vs. 20.8 months, $\mathrm{P}=0.0285$ )
(Figure 3C), whereas in the observation group, the OS of non-basal-like TNBC patients was 5.8 months longer than that of basal-like TNBC patients, although the difference was not significant (22.3 vs. 16.5 months, $\mathrm{P}=0.0658$ ) (Figure 3D). Maintenance with capecitabine significantly prolonged the OS of patients with non-basal-like TNBC (30.2 vs. 22.3 months, $\mathrm{P}=0.0257$ ) (Figure $4 A$ ), but no significant difference was observed in basal-like TNBC patients (20.8 vs. 16.5 months, $\mathrm{P}=0.0541$ ) (Figure $4 B$ ). In the maintenance group, the OS benefit was found to be present in patients over 50 years of age $(\mathrm{P}=0.005)$, in those with visceral metastases $(\mathrm{P}=0.035)$, in those with a response to initial therapy $(\mathrm{P}=0.017)$, and in those with $\mathrm{CK} 5 / 6$ - and/or EGFRnegative cancer $(\mathrm{P}=0.032)$ (Table 2). Age, visceral metastases, response to initial therapy, maintenance therapy, and CK5/6 and EGFR status were independent prognostic factors.

\section{Toxicity analysis}

In the maintenance group, 56 patients received a total of 725 cycles of chemotherapy, including 389 cycles of capecitabine maintenance therapy (median, 8 cycles), and 59 patients in the observation group underwent a total of 354 chemotherapy cycles in the absence of another 

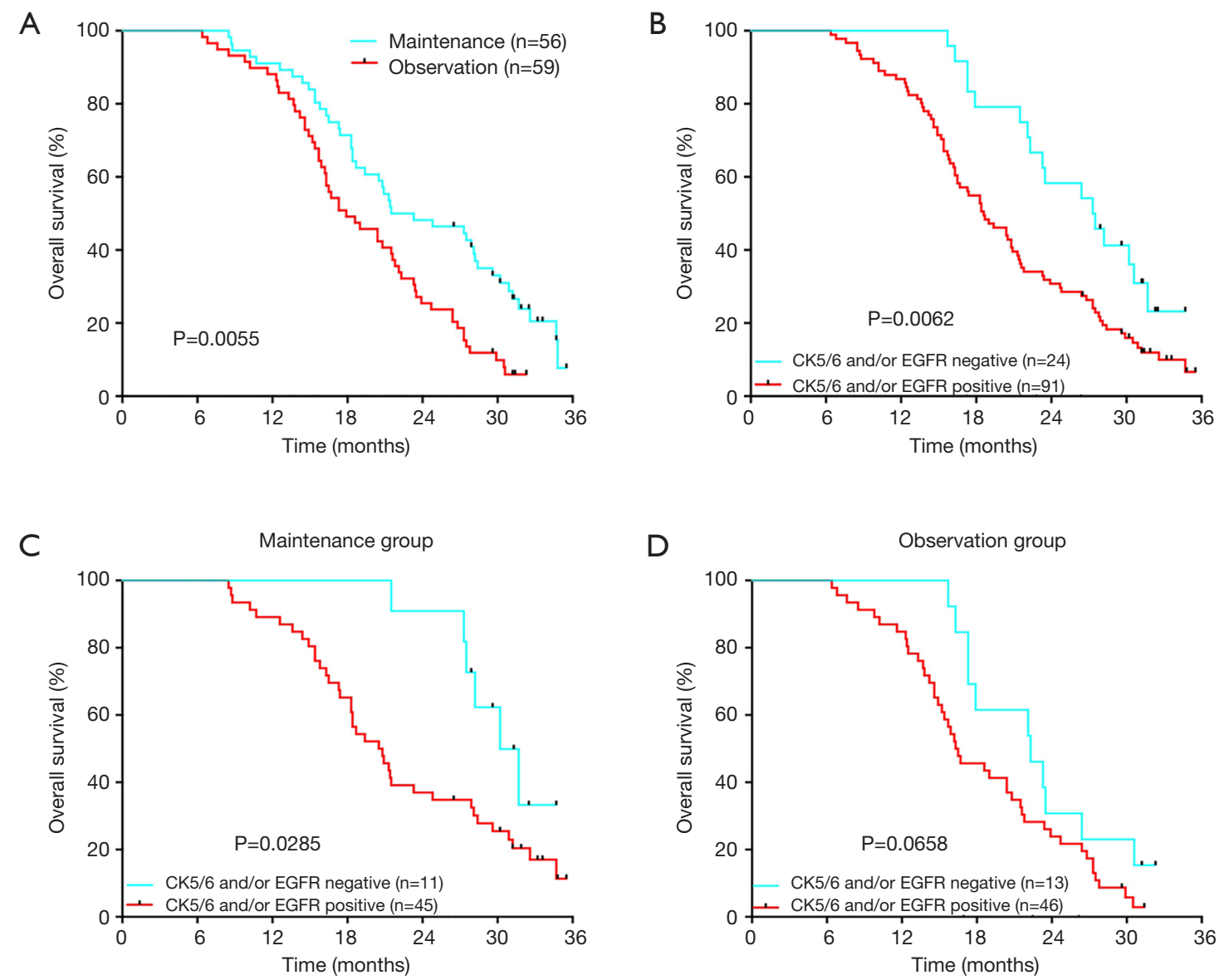

Figure 3 Overall survival (OS) of TNBC patients treated with combination chemotherapy followed by capecitabine maintenance and observation. (A) OS of the maintenance group and observation group. (B) OS of the basal-like TNBC group and non-basal-like TNBC group. (C) OS of basal-like TNBC patients and non-basal-like TNBC patients in the capecitabine maintenance group. (D) OS of basal-like TNBC patients and non-basal-like TNBC patients in the observation group. TNBC, triple-negative breast cancer.
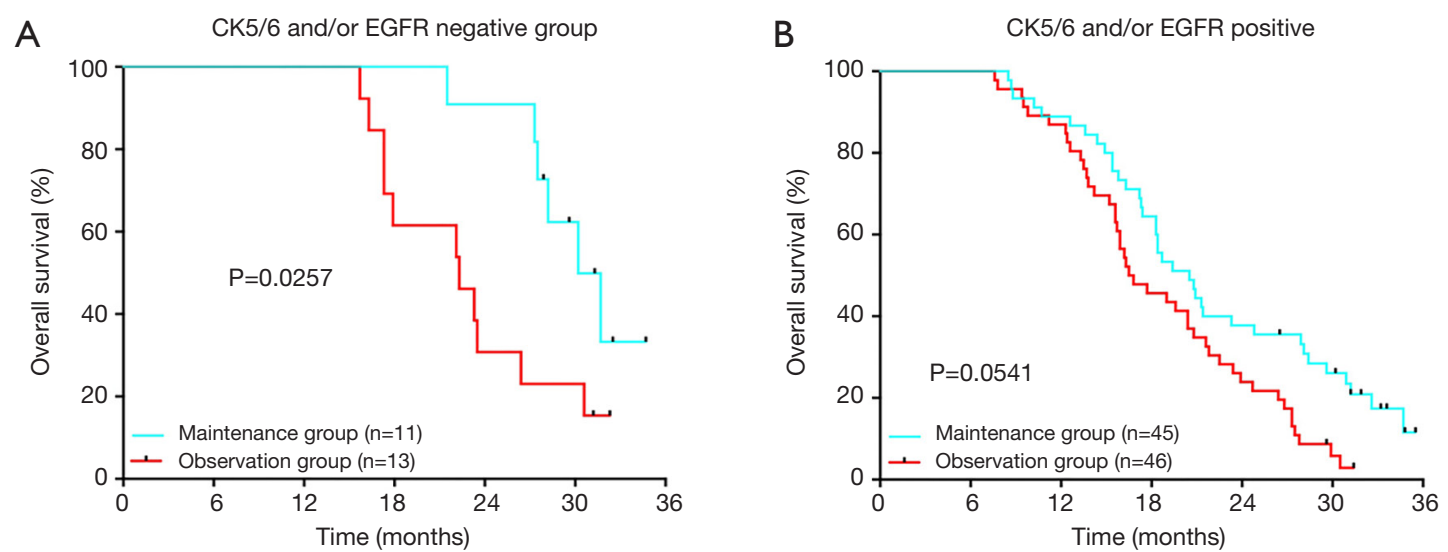

Figure 4 Overall survival (OS) in the capecitabine maintenance group and observation group. (A) OS of CK5/6- and/or EGFR-negative patients in the maintenance group and observation group. (B) OS of CK5/6- and/or EGFR positive patients in the maintenance group and observation group. EGFR, epidermal growth factor receptor. 
Table 2 Cox's proportional hazard model analysis of prognostic in patients with mTNBC

\begin{tabular}{|c|c|c|c|c|c|c|}
\hline Variables & \multicolumn{3}{|c|}{ Univariate analysis } & \multicolumn{3}{|c|}{ Multivariate analysis } \\
\hline \multicolumn{7}{|l|}{ Age (years) } \\
\hline$>50 / \leq 50$ & 0.430 & $0.255-0.723$ & 0.001 & 0.388 & $0.200-0.756$ & 0.005 \\
\hline \multicolumn{7}{|l|}{ ECOG performance status } \\
\hline \multicolumn{7}{|l|}{ Menopausal status } \\
\hline Premenopausal/postmenopausal & 1.519 & $0.787-2.648$ & 0.141 & & & \\
\hline \multicolumn{7}{|l|}{ Number of metastatic site } \\
\hline Multiple/single & 1.604 & $1.160-2.219$ & 0.004 & 1.156 & $0.751-1.781$ & 0.510 \\
\hline \multicolumn{7}{|l|}{ Response to initial therapy } \\
\hline Stable disease/response & 1.589 & $1.048-2.782$ & 0.015 & 2.076 & $1.139-3.785$ & 0.017 \\
\hline \multicolumn{7}{|l|}{ Prior adjuvant/neoadjuvant therapy } \\
\hline No/yes & 1.376 & $0.829-2.282$ & 0.217 & & & \\
\hline \multicolumn{7}{|l|}{ Group } \\
\hline Maintenance/observation & 0.337 & $0.286-0.893$ & 0.047 & 0.565 & $0.334-0.954$ & 0.033 \\
\hline \multicolumn{7}{|l|}{ CK5/6 and EGFR status } \\
\hline CK5/6 and/or EGFR negative/CK5/6 and/or EGFR positive & 0.490 & $0.250-0.960$ & 0.038 & 0.285 & $0.089-0.917$ & 0.032 \\
\hline
\end{tabular}

mTNBC, metastatic triple-negative breast cancer; HR, hazard ratio; Cl, confidence interval; ECOG, Eastern Cooperative Oncology Group performance status; EGFR, epidermal growth factor receptor.

treatment until disease progression. According to the NCI Common Terminology Criteria for Adverse Events (NCICTCAE), we found hematologic and digestive system toxic effects were the most common adverse events (Table 3). Toxicities of all grades were significantly more frequent in the maintenance group compared with the observation group (neutropenia, $85.71 \%$ vs. $25.87 \%, \mathrm{P}<0.001$; thrombocytopenia, $55.36 \%$ vs. $11.86 \%, \mathrm{P}<0.001$; anemia, $82.14 \%$ vs. $52.54 \%, \mathrm{P}=0.001$; nausea, $83.47 \%$ vs. $11.86 \%$, $\mathrm{P}<0.001$; vomiting, 69.64\% vs. $8.47 \%, \mathrm{P}<0.001$ ), and handfoot syndrome (HFS), $32.14 \%$ vs. $8.47 \%, \mathrm{P}=0.002$ ). The incidence rates of grade 3-4 neutropenia and (hand-foot syndrome) were also significantly higher in the maintenance group $(55.9 \%$ vs. $2.6 \%, \mathrm{P}<0.001 ; 8.93 \%$ vs. $0 \%, \mathrm{P}=0.019)$.

\section{Discussion}

At present, cytotoxic drugs are still the main treatment for TNBC. However, once chemotherapy is withdrawn, tumors can quickly relapse and metastasize. Several studies have reported mPFS ranging from 3.8-5.1 months after the termination of chemotherapy $(19,20)$. Therefore, maintenance therapy is particularly important, and increased attention is being focused on maintenance therapy in patients with TNBC. However, there is great variation in the mPFS (7.6-9.1 months) and mOS (18.1-19.2 months) reported by different studies $(21,22)$.

The prognosis of TNBC currently depends on the molecular subtype. Among the diverse subtypes of TNBC, basal-like is one of the most malignant. The gold standard for identifying BLBC is still based on gene expression profiling. However, technical problems and high costs limit the detection of gene expression profiles as a routine test in clinical practice. Using immunohistochemistry, numerous studies have shown that BLBC can be identified by ER-, PR-, HER-, CK5/6, and/or EGFR positivity (23-25). In 
Table 3 Treatment-related toxicities

\begin{tabular}{|c|c|c|c|c|c|c|c|c|c|c|}
\hline \multirow{2}{*}{ Adverse event } & \multicolumn{5}{|c|}{ All grades } & \multicolumn{5}{|c|}{ Grade 3-4 } \\
\hline & $\mathrm{N}$ & $\%$ & $\mathrm{~N}$ & $\%$ & $P$ & $\mathrm{~N}$ & $\%$ & $\mathrm{~N}$ & $\%$ & $P$ \\
\hline Neutropenia & 48 & 85.71 & 15 & 25.87 & $<0.001$ & 18 & 32.14 & 2 & 3.39 & $<0.001$ \\
\hline Thrombocytopenia & 31 & 55.36 & 7 & 11.86 & $<0.001$ & 3 & 5.36 & 0 & 0.00 & 0.072 \\
\hline Nausea & 42 & 83.47 & 7 & 11.86 & $<0.001$ & 3 & 5.36 & 0 & 0.00 & 0.072 \\
\hline Vomit & 39 & 69.64 & 5 & 8.47 & $<0.001$ & 2 & 3.57 & 0 & 0.00 & 0.143 \\
\hline Constipation & 15 & 26.79 & 8 & 11.86 & 0.076 & 0 & 0.00 & 0 & 0.00 & NA \\
\hline Azotemia & 3 & 5.36 & 0 & 0.00 & 0.072 & 0 & 0.00 & 0 & 0.00 & NA \\
\hline
\end{tabular}

NA, not assessable; HFS, hand-foot syndrome.

particular, CK5/6 is considered to be an extremely useful marker for identifying TNBC subtypes (26).

CK5/6 expression has been reported in $24-72 \%$ of TNBCs $(27,28)$. Patients with CK5/6-positive tumors often show a shorter survival time, and CK5/6 has been put forward as an independent prognostic factor in breast cancer $(11,26)$. The overexpression of EGFR has been observed in more than half (57\%) of BLBCs (11), and its expression was reported to be closely related to tumor grade and lymph node metastasis in $60.3-71.4 \%$ of patients with TNBC. Another study reported that patients with EGFR-positive TNBC responded poorly to neoadjuvant chemotherapy and showed poor DFS and OS compared with patients with EGFR-negative status. Furthermore, a multivariate analysis showed EGFR to be an independent predictor of PFS and OS in TNBC $(29,30)$. Thus, CK5/6 and EGFR are widely considered as molecular markers for BLBC. Using this surrogate panel, $79.13 \%$ of patients with TNBC were classified as BLBC in our study. Among the 115 patients who achieved disease control, we found that the mOS of patients with BLBC was significantly shorter than that of non-BLBC patients (18.6 vs. 27.4 months, $\mathrm{P}=0.0062$ ), and the prognosis of non-BLBC patients was significantly better than that of BLBC patients. Multivariate regression analysis revealed that age, visceral metastases, response to initial therapy, maintenance therapy, and CK5/6 and EGFR status were independent prognostic factors for, which was similar to the results reported by other studies $(3,31,32)$.

Our investigation also confirmed that CK5/6 and EGFR were biomarkers for the prognosis of TNBC patients. Maintenance therapy has been recommended for metastatic TNBC by multiple guidelines, and our study showed that after 6 cycles of initial chemotherapy, capecitabine maintenance therapy achieved improved PFS and OS in TNBC patients. Simultaneously, we also examined CK5/6 and EGFR expression to evaluate the effect of capecitabine maintenance therapy on prognosis in BLBC and non-BLBC patients. We observed that maintenance with capecitabine significantly prolonged the OS of patients with non-basallike TNBC (30.2 months vs. 22.3 months, $\mathrm{P}=0.0257)$, but there was no significant difference in the OS of basal-like TNBC patients (20.8 vs. 16.5 months, $\mathrm{P}=0.0541)$. This suggested that CK5/6 and EGFR might predict the efficacy of capecitabine maintenance therapy in TNBC.

A platinum-based combination chemotherapy regimen has been considered as an alternative or even as the preferred first-line chemotherapy option for treating patients with mTNBC (33-35). Some preliminary clinical trial data (36), a randomized neoadjuvant clinical study (37), and a retrospective case review of adjuvant therapy (21) have provided some level of evidence for platinum activity in TNBC patients. In our study, we observed that the objective response rate and the disease control rate were $51.83 \%$ $(85 / 164)$ and $70.12 \%(115 / 164)$ in patients with TNBC who received 6 cycles of combination chemotherapy as a firstline treatment, which was similar to the results reported by previous studies. Patients in the maintenance group received 6 cycles of combination chemotherapy and another 
8 (median) cycles of single capecitabine, while those in the observation group received only 6 cycles of combination chemotherapy. In the maintenance group, the mPFS and mOS were 1.6 and 4.5 months longer, respectively, than those in the observation group $(7.3$ vs. 5.7 months and 22.4 vs. 17.9 months, respectively), and the differences were significant. We suspect that these results are attributable to the additional maintenance treatment. More cycles may improve the clinical benefit, but more cycles also encompass more side effects $(16,20,38,39)$. Significantly higher bone marrow toxic effects, especially grade 3-4 neutropenia, were observed in the maintenance treatment group than in the observation group $(32.14 \%$ vs. $3.39 \%, \mathrm{P}<0.001)$, and the incidence rates of nausea, vomiting, and HFS were also all significantly higher in the maintenance group $(83.47 \% \mathrm{vs}$. 11.86, $\mathrm{P}<0.001 ; 69.64 .6 \%$ vs. $8.47 \%, \mathrm{P}<0.001$; and $32.14 \%$ vs. $8.47 \%, \mathrm{P}=0.002$, respectively).

In conclusion, our results confirmed that capecitabine maintenance therapy can prolong the mPFS and mOS of patients with mTNBC. This is a widely accepted therapeutic strategy for mTNBC patients. The toxic effects of the maintenance therapy were well tolerated, and the long-term clinical outcomes were encouraging. Moreover, we demonstrated that patients with non-basal-like TNBC had a better prognosis than those with basal-like TNBC and could benefit from maintenance therapy with capecitabine. Our findings suggest that CK5/6 and EGFR may serve as prognostic biomarkers in patients with TNBC and could be used to predict the efficacy of capecitabine maintenance therapy.

\section{Acknowledgments}

The authors thank B. Draper and J. Reynolds for English language editing.

Funding: This research was supported by the National Natural Science Foundation of China (No. 81802651) and Foundation of "Peak" Training Program for Scientific Research of Yijishan Hospital, Wannan Medical College (No. GF2019G11).

\section{Footnote}

Reporting Checklist: The authors have completed the REMARK reporting checklist. Available at http://dx.doi. org/10.21037/tcr-20-1760

Data Sharing Statement: Available at http://dx.doi. org/10.21037/tcr-20-1760

Conflicts of Interest: All authors have completed the ICMJE uniform disclosure form (available at http://dx.doi. org/10.21037/tcr-20-1760). The authors have no conflicts of interest to declare.

Ethical Statement: The authors are accountable for all aspects of the work in ensuring that questions related to the accuracy or integrity of any part of the work are appropriately investigated and resolved. The study was conducted in accordance with the Declaration of Helsinki (as revised in 2013). The study was approved by the Ethical Committee of the First Affiliated Hospital of Wannan Medical College (No.: 2008-7) and individual consent for this retrospective analysis was waived.

Open Access Statement: This is an Open Access article distributed in accordance with the Creative Commons Attribution-NonCommercial-NoDerivs 4.0 International License (CC BY-NC-ND 4.0), which permits the noncommercial replication and distribution of the article with the strict proviso that no changes or edits are made and the original work is properly cited (including links to both the formal publication through the relevant DOI and the license). See: https://creativecommons.org/licenses/by-nc-nd/4.0/.

\section{References}

1. Cardoso F, Harbeck N, Fallowfield L, et al. Locally recurrent or metastatic breast cancer: ESMO Clinical Practice Guidelines for diagnosis, treatment and followup. Ann Oncol 2012;23 Suppl 7:vii11-9.

2. O'Shaughnessy J. Extending survival with chemotherapy in metastatic breast cancer. Oncologist 2005;10 Suppl $3: 20-9$.

3. Foulkes WD, Smith IE, Reis-Filho JS. Triple-negative breast cancer. N Engl J Med 2010;363:1938-48.

4. Beslija S, Bonneterre J, Burstein H, et al. Second consensus on medical treatment of metastatic breast cancer. Ann Oncol 2007;18:215-25.

5. Cardoso F, Bedard PL, Winer EP, et al. International guidelines for management of metastatic breast cancer: combination vs sequential single-agent chemotherapy. J Natl Cancer Inst 2009;101:1174-81.

6. Xu Y, Sun Q. Headway in resistance to endocrine therapy in breast cancer. J Thorac Dis 2010;2:171-7.

7. Dent R, Trudeau M, Pritchard KI, et al. Triple-negative 
breast cancer: clinical features and patterns of recurrence. Clin Cancer Res 2007;13:4429-34.

8. Kassam F, Enright K, Dent R, et al. Survival outcomes for patients with metastatic triple-negative breast cancer: implications for clinical practice and trial design. Clin Breast Cancer 2009;9:29-33.

9. Lehmann BD, Bauer JA, Chen X, et al. Identification of human triple-negative breast cancer subtypes and preclinical models for selection of targeted therapies. J Clin Invest 2011;121:2750-67.

10. Sørlie T, Perou CM, Tibshirani R, et al. Gene expression patterns of breast carcinomas distinguish tumor subclasses with clinical implications. Proc Natl Acad Sci U S A 2001;98:10869-74.

11. Nielsen TO, Hsu FD, Jensen K, et al. Immunohistochemical and clinical characterization of the basal-like subtype of invasive breast carcinoma. Clin Cancer Res 2004;10:5367-74.

12. Rakha EA, Elsheikh SE, Aleskandarany MA, et al. Triplenegative breast cancer: distinguishing between basal and nonbasal subtypes. Clin Cancer Res 2009;15:2302-10.

13. Joensuu H, Kellokumpu-Lehtinen PL, Huovinen R, et al. Adjuvant Capecitabine in Combination With Docetaxel, Epirubicin, and Cyclophosphamide for Early Breast Cancer: The Randomized Clinical FinXX Trial. JAMA Oncol 2017;3:793-800.

14. Li J, Yu K, Pang D, et al. Adjuvant Capecitabine With Docetaxel and Cyclophosphamide Plus Epirubicin for Triple-Negative Breast Cancer (CBCSG010):An OpenLabel, Randomized, Multicenter, Phase III Trial. J Clin Oncol 2020;38:1774-84.

15. Lluch A, Barrios CH, Torrecillas L, et al. Phase III Trial of Adjuvant Capecitabine After Standard Neo-/Adjuvant Chemotherapy in Patients With Early Triple-Negative Breast Cancer (GEICAM/2003-11_CIBOMA/2004-01). J Clin Oncol 2020;38:203-13.

16. Ferrero JM, Hardy-Bessard AC, Capitain O, et al. Weekly paclitaxel, capecitabine, and bevacizumab with maintenance capecitabine and bevacizumab as first-line therapy for triple-negative, metastatic, or locally advanced breast cancer: Results from the GINECO A-TaXel phase 2 study. Cancer 2016;122:3119-26.

17. Liang X, Di L, Song G, et al. Capecitabine maintenance therapy for XT chemotherapy-sensitive patients with metastatic triple-negative breast cancer. Chin J Cancer Res 2014;26:550-7.

18. Eisenhauer EA, Therasse P, Bogaerts J, et al. New response evaluation criteria in solid tumours: revised RECIST guideline (version 1.1). Eur J Cancer 2009;45:228-47.

19. Alba E, Ruiz-Borrego M, Margelí M, et al. Maintenance treatment with pegylated liposomal doxorubicin versus observation following induction chemotherapy for metastatic breast cancer: GEICAM 2001-01 study. Breast Cancer Res Treat 2010;122:169-76.

20. Park YH, Jung KH, Im SA, et al. Phase III, multicenter, randomized trial of maintenance chemotherapy versus observation in patients with metastatic breast cancer after achieving disease control with six cycles of gemcitabine plus paclitaxel as first-line chemotherapy: KCSGBR07-02. J Clin Oncol 2013;31:1732-9.

21. Harper NW, Hodges KB, Stewart RL, et al. Adjuvant Treatment of Triple-Negative Metaplastic Breast Cancer With Weekly Paclitaxel and Platinum Chemotherapy: Retrospective Case Review From a Single Institution. Clin Breast Cancer 2019;19:e495-500.

22. Symonds L, Linden H, Gadi V, et al. Combined Targeted Therapies for First-line Treatment of Metastatic Triple Negative Breast Cancer-A Phase II Trial of Weekly NabPaclitaxel and Bevacizumab Followed by Maintenance Targeted Therapy With Bevacizumab and Erlotinib. Clin Breast Cancer 2019; 19:e283-96.

23. Cheang MC, Voduc D, Bajdik C, et al. Basal-like breast cancer defined by five biomarkers has superior prognostic value than triple-negative phenotype. Clin Cancer Res 2008;14:1368-76.

24. Lachapelle J, Foulkes WD. Triple-negative and basal-like breast cancer: implications for oncologists. Curr Oncol 2011;18:161-4.

25. Livasy CA, Karaca G, Nanda R, et al. Phenotypic evaluation of the basal-like subtype of invasive breast carcinoma. Mod Pathol 2006;19:264-71.

26. Abd El-Rehim DM, Pinder SE, Paish CE, et al. Expression of luminal and basal cytokeratins in human breast carcinoma. J Pathol 2004;203:661-71.

27. Dogu GG, Ozkan M, Ozturk F, et al. Triple-negative breast cancer: immunohistochemical correlation with basaloid markers and prognostic value of survivin. Med Oncol 2010;27:34-9.

28. Ryu DW, Jung MJ, Choi WS, et al. Clinical significance of morphologic characteristics in triple negative breast cancer. J Korean Surg Soc 2011;80:301-6.

29. Abdelrahman AE, Rashed HE, Abdelgawad M, et al. Prognostic impact of EGFR and cytokeratin 5/6 immunohistochemical expression in triple-negative breast cancer. Ann Diagn Pathol 2017;28:43-53.

30. Zhang M, Zhang X, Zhao S, et al. Prognostic value 
of survivin and EGFR protein expression in triplenegative breast cancer (TNBC) patients. Target Oncol 2014;9:349-57.

31. Chen MH, Yip GW, Tse GM, et al. Expression of basal keratins and vimentin in breast cancers of young women correlates with adverse pathologic parameters. Mod Pathol 2008;21:1183-91.

32. Yue Y, Astvatsaturyan K, Cui X, et al. Stratification of Prognosis of Triple-Negative Breast Cancer Patients Using Combinatorial Biomarkers. PLoS One 2016;11:e0149661.

33. Elserafi MM, Zeeneldin AA, Abdelsalam IM, et al. First-line paclitaxel and cisplatin used sequentially or in combination in metastatic breast cancer: A phase II randomized study. J Egypt Natl Canc Inst 2018;30:13-20.

34. Fan $\mathrm{Y}, \mathrm{Xu} \mathrm{BH}$, Yuan $\mathrm{P}$, et al. Docetaxel-cisplatin might be superior to docetaxel-capecitabine in the first-line treatment of metastatic triple-negative breast cancer. Ann Oncol 2013;24:1219-25.

35. Zhang J, Wang Z, Hu X, et al. Cisplatin and gemcitabine as the first line therapy in metastatic triple negative breast cancer. Int J Cancer 2015;136:204-11.

36. Leong CO, Vidnovic N, DeYoung MP, et al. The p63/

Cite this article as: Zhu Y, Li K, Zhang J, Wang L, Sheng L, Yan L. The prognostic and predictive significance of cytokeratin $5 / 6$ and epidermal growth factor receptor in metastatic triplenegative breast cancer treated with maintenance capecitabine. Transl Cancer Res 2021;10(3):1193-1203. doi: 10.21037/tcr-20-1760 p73 network mediates chemosensitivity to cisplatin in a biologically defined subset of primary breast cancers. J Clin Invest 2007;117:1370-80.

37. Sikov WM, Berry DA, Perou CM, et al. Impact of the addition of carboplatin and/or bevacizumab to neoadjuvant once-per-week paclitaxel followed by dosedense doxorubicin and cyclophosphamide on pathologic complete response rates in stage II to III triple-negative breast cancer: CALGB 40603 (Alliance). J Clin Oncol 2015;33:13-21.

38. Dong G, Jia Y, Wang X, et al. The comparison of maintenance treatment with capecitabine (CMT) and nonmaintenance treatment with capecitabine (non-CMT) in patients with metastatic breast cancer. Int J Clin Exp Med 2015;8:8283-7.

39. Gligorov J, Doval D, Bines J, et al. Maintenance capecitabine and bevacizumab versus bevacizumab alone after initial first-line bevacizumab and docetaxel for patients with HER2-negative metastatic breast cancer (IMELDA): a randomised, open-label, phase 3 trial. Lancet Oncol 2014;15:1351-60. 